\title{
The Association of Obesity with Walking and Balance Control in Fully Ambulatory People with Multiple Sclerosis According to Two Different Classifications
}

\author{
(1) Asiye Tuba Ozdogar1, (1) Turhan Kahraman², (1) Ozge Ertekin33, (10 Cavid Baba4, (10 Serkan Ozakbas4 \\ 1Dokuz Eylul University, Graduate School of Health Sciences, Izmir, Turkey \\ 2lzmir Katip Celebi University Faculty of Health Sciences, Department of Physiotherapy and Rehabilitation, Izmir, Turkey \\ ${ }^{3}$ Dokuz Eylül University, School of Physical Therapy and Rehabilitation, Izmir, Turkey \\ 4Dokuz Eylul University Faculty of Medicine, Department of Neurology, Izmir, Turkey
}

\section{Abstract}

Objective: Previous studies have reported that people with obesity have slower walking speed, poor balance control, and more energy expenditure during gait than patients without obesity. However, little is known about the effect of obesity on walking and balance control in people with multiple sclerosis (MS). This study aimed to investigate the associations of obesity with walking and balance control in fully ambulatory people with MS using two different obesity classifications.

Materials and Methods: This study included 210 fully ambulatory people with MS. Obesity classification recommended for MS and obesity classification by the World Health Organization (WHO) were used. Outcome measures included walking speed [timed 25-foot walk (T25FW)], walking endurance [six-minute walk test $(6 \mathrm{MWT})$ ], perceived walking impairment [12-item ms walking scale (MSWS-12)], and balance control [timed up and go (TUG) test].

Results: According to recommendations for MS and WHO classification, 105 (50\%) and 28 (13.3\%) participants were classified as obese, respectively. Both groups revealed that patients who are overweight and obese have lower scores in T25FW, 6MWT, and TUG tests, whereas higher scores on the MSWS-12 than patients without obesity, with a significant difference $(p<0.05)$.

Conclusion: People who are overweight and obese with MS have poorer performance on walking speed, walking endurance, perceived walking impairment, and balance control than non-obese counterparts. Future longitudinal studies should investigate the effects of losing weight on walking and balance in people with MS.

Keywords: Multiple sclerosis, obesity, walking, balance

\section{Introduction}

Multiple sclerosis (MS) is a demyelinating and progressive disease of the central nervous system caused by genetic, lifestyle, and environmental factors (1). Obesity is one of the risk factors for developing MS. Elevated body mass index (BMI) has been associated with a younger age at MS onset and a higher risk for developing MS (2). Additionally, recent research has reported that obesity is associated with greater neuroinflammation, relapse risk, and disability progression (3). Obesity also has adverse effects on depression, functional capacity, and healthrelated quality of life in people with MS $(4,5)$. Study reports on the prevalence of obesity in MS and the general population are inconsistent (5-7).

Balance problems are common symptoms in MS, even early in the disease course (8). Balance and gait dysfunction is present in $50 \%-80 \%$ of people with MS, and over $50 \%$ of patients reported a falling episode at least once a year (8). Of those with mobility impairments, $70 \%$ described it as the most

Address for Correspondence: Asiye Tuba Ozdogar, Dokuz Eylul University, Graduate School of Health Sciences, Izmir, Turkey E-mail: asiye.tuba.ozdogar@gmail.com ORCID-ID: orcid.org/0000-0003-0043-9374 Received: 05.09.2021 Accepted: 04.12.2021

'Copyright 2021 by the Journal of Multiple Sclerosis Research published by Galenos Publishing House. 
challenging symptom (9). Obesity is also a risk factor for poor balance and walking difficulties in MS in addition to muscle weakness, cerebellar ataxia, abnormal tone, sensory loss, visual impairments, cognitive impairment, neuropathic pain, and fatigue $(9,10)$.

Kalron (11) reported significant differences in walking speed, step length, step width, and double support period in patients with MS and obesity compared to those with MS and normal weight. Contrarily, Pilutti et al. (10) found no significant difference in various mobility outcomes, such as walking speed and endurance, based on the BMI status. Sebastião et al. (12) showed that patients with MS and obesity have lower cardiorespiratory fitness than overweight and non-obese groups. Studies that investigate the relationship between the lower limb functions and obesity in people with MS have presented mixed results. However, data to understand the effects of obesity on walking and balance control in fully ambulatory people with MS is insufficient.

Two different methods are presented for the classification of obesity in MS. The first one is the standard way that was developed by the World Health Organization (WHO) (13), the second one was recommended by Pilutti and Motl (14). However, no consensus was determined on which to use in MS. Thus, this study aimed to investigate the effect of obesity on walking and balance control in a large sample of fully ambulatory people with MS using two different obesity classifications.

\section{Materials and Methods}

\section{Study Design}

This cross-sectional study was conducted at the MS Center of Dokuz Eylul University. The baseline data of continuing research entitled, "Follow-up of physical, psychosocial, and cognitive influences in people with MS: a prospective cohort study" (ClinicalTrials.gov Identifier: NCT03878836) was used in the study and approved by the Dokuz Eylul University Ethics Committee (approval number: 2021/12-34 and protocol number: 2959-GOA). The study was conducted following the principles of the Declaration of Helsinki (as revised in Brazil 2013). Written consent was obtained from all participants.

\section{Participants}

The inclusion criteria were having MS based on the 2017 McDonald's criteria (15), ability to walk at least $20 \mathrm{~m}$ without resting, and a relapse-free period of 30 days. Exclusion criteria were neurological disorder other than MS; relapse throughout the study period; history of orthopedic surgery including ankle-foot, knee, hip, or spine that influences balance and walking. Baseline data of participants were extracted from the registry database [iMed (version 6.8; Merck Serono SA, Geneva, Switzerland)] from October 2016 to October 2020. The data used in these analyses were from our ongoing study that included patients who started or undergoing diseasemodifying therapies changes, thus all participants had a relapsing-remitting type of MS.

Obesity classification included two methods, the one recommended by Pilutti and Motl (14). and the WHO classification. Using the recommended method, participants with a BMI of $>24.7 \mathrm{~kg} / \mathrm{m}^{2}$ (this cut-off score has been shown as the best threshold that distinguishes body fat-defined obesity in MS) were classified as people with obesity (14). According to the WHO classification, participants were divided into three groups as normal weight $\left(<25.0 \mathrm{~kg} / \mathrm{m}^{2}\right)$, overweight (25.0-29.9 $\left.\mathrm{kg} / \mathrm{m}^{2}\right)$, and obese $\left(>30.0 \mathrm{~kg} / \mathrm{m}^{2}\right)(13)$.

\section{Outcome Measures}

Demographic and clinical characteristics (gender, age, MS course, and disease duration) were used as baseline data.

The expanded disability status scale (EDSS) scoring, as the universal measure to the disability level in people with $\mathrm{MS}$, is performed according to the neurological examination of seven functional systems (cerebellar, pyramidal, sensory, brainstem, bladder and bowel, visual, and cerebral). The ambulatory state of the patients is also recorded (16). All patients were examined by the same senior MS neurologist, who also calculated the EDSS scores.

The walking speed was assessed by the timed 25 -foot walk (T25FW). The T25FW is the best-defined and reliable method to evaluate walking disability in people with MS. Participants were asked to walk in $7.62 \mathrm{~m}$ (25 feet) as fast and safely as possible. Time is recorded in seconds. The task was performed twice, and the T25FW score was calculated as the average time of two trials (17).

The six-minute walk test (6MWT) was used to assess walking endurance. The standard guidelines were used while performing the 6MWT. Distance (meters) covered in 6 min was reported. A higher score indicates higher walking endurance (18).

The timed and up go (TUG) test is an objective, reliable, and simple measurement method to evaluate balance and functional mobility. During the test, the participant was asked to stand up from a standard chair, walk $3 \mathrm{~m}$, turn around, walk back to the chair, and sit. The score is the time (seconds) to perform the test (19).

The multiple sclerosis walking scale-12 (MSWS-12) was developed to assess the walking impairment that is perceived by people with MS, which consists of 12 questions with Likert-type answers. A higher score indicates poorer walking performance (20). The valid Turkish version of the MSWS-12 was used (21).

\section{Data Analysis}

Normal distribution of data was checked using the KolmogorovSmirnov test and histograms. Box plots were used to define the 
outlier values for each outcome. The comparison between the groups was performed using an independent-samples t-test for the continuous variables, and the chi-square test for the categorical variables. The multivariate analysis of covariance test was performed to test the differences between the groups. Covariates included age, sex, and the EDSS score. Assumptions for multivariate analysis of covariance were reached according to matrix scatter plots and the Wilk $\lambda$ test. Partial spearman's correlation coefficients were investigated between the BMI and walking and balance parameters while controlling for EDSS. Statistical significance was set at $p$-values of $<0.05$. Data were analyzed using the International Business Machines ${ }^{\circledR}$ Statistical Package for the Social Sciences ${ }^{\circledR}$ Statistics software (Version 25.0. Armonk, NY: IBM Corp.).

\section{Results}

The data analysis included 210 people with MS. According to the classification that was recommended by Pilutti and Motl (14) (50\%) were classified as obese. No significant difference was observed between the groups regarding gender and disease duration ( $p>0.05$ ). A significant difference was found in age, disability level, and BMI between patients with obesity and without $(p<0.05)$ (Table 1).
Using the WHO criteria, 28 (13.33\%) were classified as obese. Significant differences were observed regarding age, gender, disability level, and BMI between the groups (normal weight, overweight, and obese) $(p<0.05)$. Disease duration was not significantly different ( $p>0.05$ ) (Table 2).

Patients in both classification methods significantly differed in the T25FW, 6MWT, TUG, and MSWS-12 scores with small to medium effect sizes $(p<0.05)$. Table 3 and scatter plots (Figure 1-4) present the detailed comparisons of walking speed, walking endurance, perceived walking impairment, and balance control between the groups. A significant weak correlation was found between the BMI and walking and balance parameters (Table 4).

\section{Discussion}

The current study has indicated that obesity is associated with slower walking endurance, walking speed, and balance control, and higher perceived walking impairment in people with MS regardless of the BMI classification methods. The lower limit for obesity based on the classification by Pilutti and Motl (14). is a BMI of $>24.7 \mathrm{~kg} / \mathrm{m}^{2}$. With this cut-off, half of our participants fall into the category of obesity. Our data were compared with the general population to evaluate the frequency. In this context,

Table 1. Demographic and clinical characteristics of participants (according to the recommended classification)

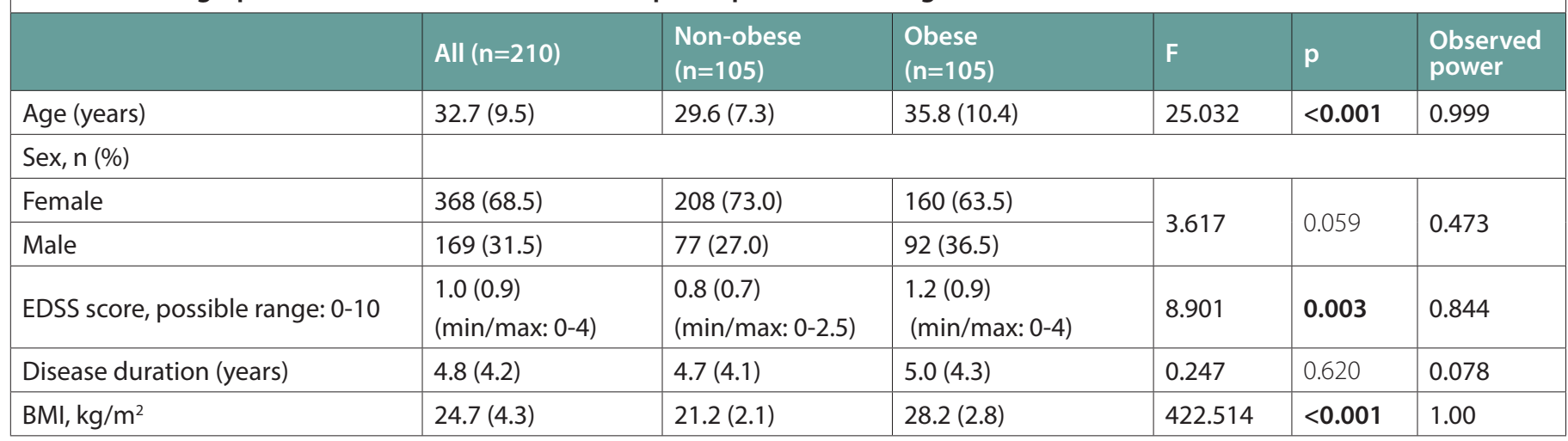

Significant $\mathrm{p}$-values are presented in bold. Values are presented as mean (SD) unless specified. EDSS: Expanded disability status scale, BMI: Body mass index

Table 2. Demographic and clinical characteristics of participants (according to the WHO classification)

\begin{tabular}{|c|c|c|c|c|c|c|}
\hline & $\begin{array}{l}\text { Non-obese } \\
(n=113)\end{array}$ & Overweight (n=69) & Obese $(n=28)$ & $\mathbf{F}$ & p & $\begin{array}{l}\text { Observed } \\
\text { power }\end{array}$ \\
\hline Age (years) & $30.038(7.84)$ & $36.16(11.30)$ & $33.79(8.20)$ & 8.734 & $<0.001$ & 0.969 \\
\hline Female & $91(80.5)$ & $44(63.8)$ & $21(75.0)$ & \multirow{2}{*}{3.207} & \multirow{2}{*}{0.043} & \multirow{2}{*}{0.608} \\
\hline Male & $22(19.5)$ & $25(36.2)$ & $7(25.0)$ & & & \\
\hline Disease duration (years) & $4.76(4.13)$ & $4.80(4.50)$ & $5.15(3.58)$ & 0.102 & 0.903 & 0.065 \\
\hline $\mathrm{BMI}, \mathrm{kg} / \mathrm{m}^{2}$ & $21.46(2.24)$ & $27.03(1.31)$ & $32.07(1.73)$ & 419.132 & $<0.001$ & 1.00 \\
\hline
\end{tabular}

Significant $\mathrm{p}$-values are presented in bold. Values are presented as mean (SD) unless specified. EDSS: Expanded disability status scale, BMI: Body mass index 


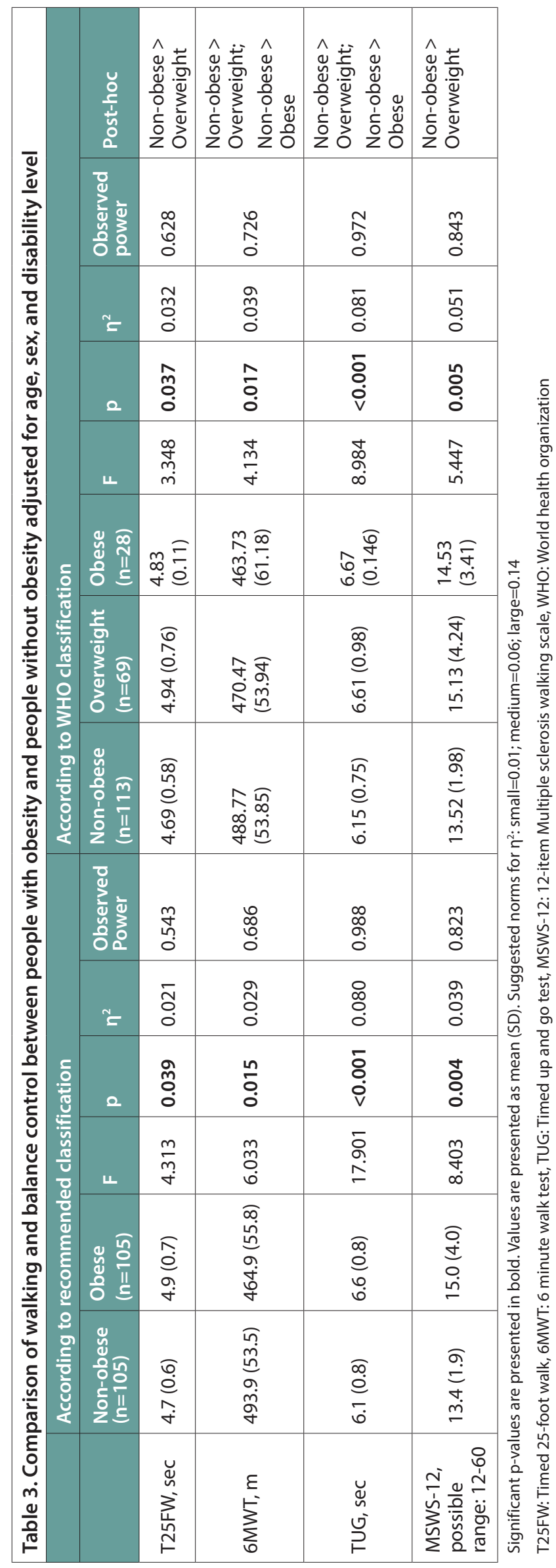

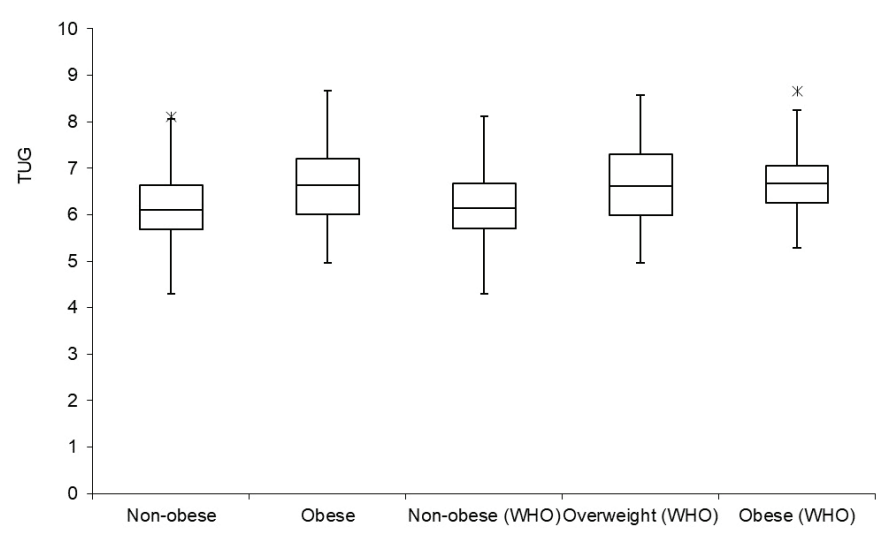

Figure 1. Comparison of balance control between the groups WHO: World health organization

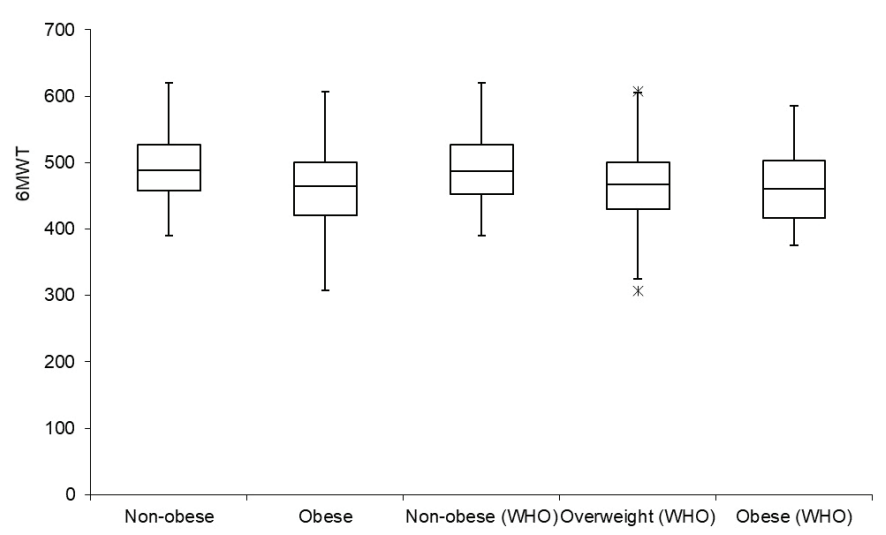

Figure 2. Comparison of walking endurance between the groups WHO: World health organization

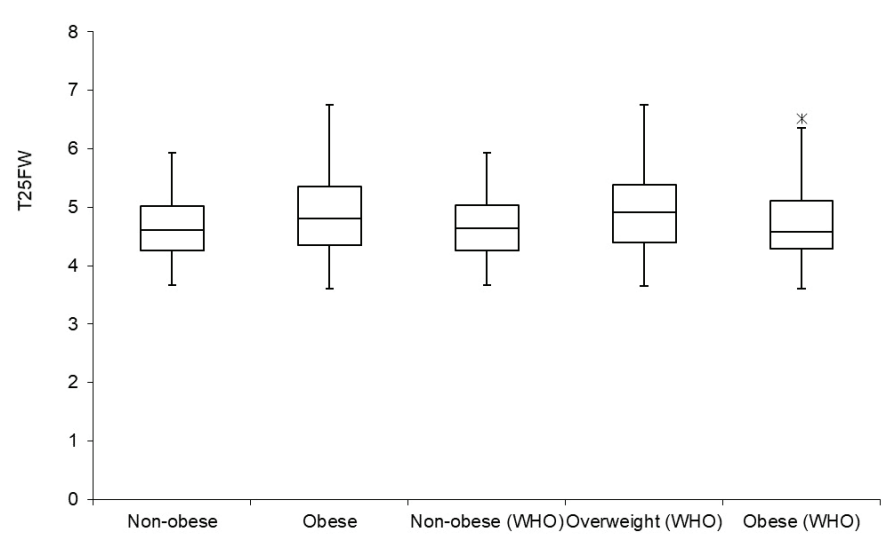

Figure 3. Comparison of walking speed between the groups WHO: World health organization

the limit used to determine obesity in the MS population is approximately the limit of "overweight" compared to the WHO cut-off scores for the general population. The prevalence of overweight and obesity has been found in $65.05 \%$ of women and $58 \%$ of men in the general Turkish population using the WHO classification. Our study revealed that $41.7 \%$ of women 


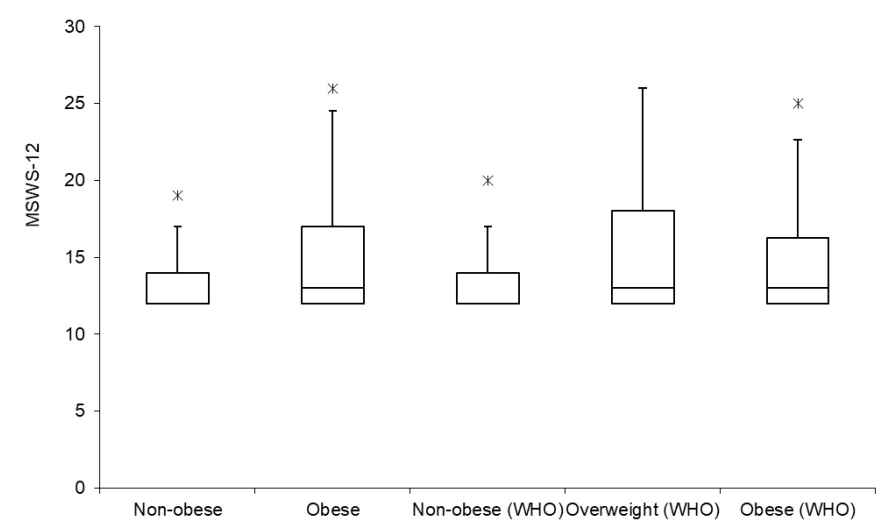

Figure 4. Comparison of perceived walking impairment between the groups

WHO: World health organization

\begin{tabular}{|c|c|c|c|c|}
\hline & \multicolumn{4}{|c|}{ Body mass index } \\
\hline & \multicolumn{2}{|c|}{$\begin{array}{l}\text { Spearman's } \\
\text { correlation }\end{array}$} & \multicolumn{2}{|c|}{$\begin{array}{l}\text { Partial } \\
\text { correlation }{ }^{\mathrm{a}}\end{array}$} \\
\hline & r & p & r & $p$ \\
\hline T25FW & 0.164 & 0.017 & 0.181 & 0.009 \\
\hline TUG & 0.293 & $<0.001$ & 0.280 & $<0.001$ \\
\hline $6 \mathrm{MWT}$ & -0.239 & $<0.001$ & -0.235 & 0.001 \\
\hline MSWS-12 & 0.183 & 0.008 & 0.117 & 0.010 \\
\hline
\end{tabular}

aAdjusted for EDSS, Significant p-values are presented in bold. BMI: Body mass index, T25FW: Timed 25-foot walk, 6MWT: 6 minute walk test, TUG: Timed up and go test, MSWS-12: 12-item Multiple sclerosis walking scale

and $59.3 \%$ of men were classified as overweight and obese (22). Obesity frequency seems similar in men; however, women with MS have less frequency than the general population.

A recently published review on the comorbidities in MS emphasized that obesity and overweight are associated with longer diagnostic delays, more rapid disability progression, and increased cardiac risk (23). Additionally, Marck et al. (24) showed that overweight and obese people with MS reported a worse quality of life in terms of mental and physical health than those with normal weight. Considering the high prevalence and adverse impact of obesity on the course of MS, patient care, physiological status, and systemic and pulmonary complications, evaluating obesity in MS is of worth $(25,26)$.

Similarly, Kalron (11) examined the obesity in people with MS using $24.7 \mathrm{~kg} / \mathrm{m}^{2}$ as a cut-off value and found that the obese group walked slower and had shorter step lengths, wider step width, and prolonged double support period than non-obese subjects. Additionally, the obese group had shorter distances and poor balance performance on clinical measures. However, no significant differences were found between the groups in MSWS-12 and static postural control assessment that was evaluated with Zebris FDM-T Treadmill. They hypothesized that obesity could have negatively affected the dynamic activities, such as gait, rather than static balance activities (11). This study used the TUG test to evaluate the dynamic balance. Therefore, our results also support this hypothesis.

Furthermore, Pilutti et al. (10) investigated the effects of weight on several mobility parameters, such as gait kinematics and selfreported walking impairment, by classifying the participants into four groups as normal weight, overweight, and obese classes 1 and 2. No significant relationship was found between the weight status and mobility assessments (10). The "obese class 2 " group was not included in our study because no BMI value of $>35.0 \mathrm{~kg} / \mathrm{m}^{2}$ was recorded. Therefore, walking endurance and balance control was significantly affected in overweight and obese groups compared to non-obese people with MS. However, walking speed and perceived walking impairment were not significantly different between normal and obese groups. Using two classification methods to determine obesity could be the main reason for conflicting results. The low prevalence of obesity in our MS population according to the WHO classification could also add to this discrepancy.

Our study revealed a 1.0 mean EDSS score. However, Liparoti et al. (27) showed that walking impairments could arise from the presence of cognitive impairment, fatigue, and depression in people with MS without any clinically detectable walking abnormalities. Additionally, Ayan et al. (28) reported that people with MS with the absence of clinical disability have a low balance and gait performance compared to the healthy controls. Moon et al. (29) investigated the walking changes in people with MS with minimal disability and compared it with healthy controls using a temporal-spatial gait analysis (GAITRite system). They revealed that walking speed, step features (e.g., length and width), and double support time were affected people with MS with minimal disability compared to the controls (29). This finding makes us think that walking impairments and balance problems can occur even in people with lower levels of disability, and higher BMI could be one of the risk factors.

Furthermore, studies that investigate the effects of obesity in the general population reported possible compensator alterations of gait mechanics, such as decreased walking speed, prolate double support, and stance times, in the obese and overweight subjects (30). Manawat and Shweta (31) compared obese people with non-obese to determine the relationship between the BMI and 6MWT and revealed that increases in BMI induce decreases in 6MWT performance. Likewise, a negative correlation was detected between the BMI and 6MWT, whereas a positive correlation between the BMl and walking speed, perceived walking impairment, and balance control.

In the clinic aspect, many studies have investigated the effects of different types of exercise modalities to improve walking and balance; however, little is known about the management of obesity that could positively affect walking and balance 
performance (32,33). Mokhtarzade et al. (34) reported that elevated body composition might adversely influence the progression, course, and treatment in the MS progression, which is directly related to gait and balance. Additionally, Pilutti et al. (35) provided some evidence about the efficacy of 6-month internet-delivered physical activity behavioral intervention on body composition. A systematic review demonstrated that aerobic and strength exercises are the essential parts of dealing with obesity in the general population; however, even the most effective intensity and type of exercise are still unclear (36). Therefore, body composition should be considered when prescribing exercise for walking impairment and balance problems in people with MS.

Strengths of this research include the large sample size that increases the study's power and the low level of disability of participants that eliminated other factors that affect walking and balance in MS. The study has limitations in the used measurement methods. BMI used to identify obesity is cost effective and convenient to use. However, it does not provide information about people's adiposity. Clinical measurement technics were used for walking and balance assessment. Laboratory-based measurement could provide more detailed information on the characteristics of walking and balance. Another limitation is the absence of a healthy control group, which could help us understand the differences in walking that arise from obesity that are specific to people with MS.

\section{Conclusion}

This study revealed that people who are overweight and obese with MS have less walking speed, walking endurance, perceived walking impairment, and balance control than nonobese counterparts regardless of the BMI classification method. Moreover, we speculate that being overweight and obese could be important factors affecting walking and balance in people with mild disability in MS. Future studies can provide answers to the management of obesity via exercise programs to improve walking and balance control in people with MS.

\section{Acknowledgments}

The authors acknowledge the Multiple Sclerosis Research Association for assistance during the recruitment of the study.

\section{Ethics}

Ethics Committee Approval: The study and approved by the Dokuz Eylul University Ethics Committee (approval number: 2021/12-34 and protocol number: 2959-GOA).

Informed Consent: Written consent was obtained from al participants.

\section{Authorship Contributions}

Surgical and Medical Practices: C.B. S.O., Concept: A.T.O., T.K., O.E., C.B., S.O., Design: A.T.O., T.K., O.E., C.B., S.O., Data Collection or Processing: A.T.O., Analysis or Interpretation: A.T.O., T.K., Literature Search: A.T.O., T.K., O.E., C.B., S.O., Writing: A.T.O., T.K., O.E., C.B., S.O.

Conflict of Interest: No conflict of interest was declared by the authors.

Financial Disclosure: The authors declared that this study received no financial support.

\section{REFERENCES}

1. Hunter SF. Overview and diagnosis of multiple sclerosis. Am J Manag Care 2016;22(Suppl 6):s141-s150.

2. Mokry LE, Ross S, Timpson NJ, Sawcer S, Davey Smith G, Richards JB. Obesity and Multiple Sclerosis: A Mendelian Randomization Study. PLoS Med 2016;13:e1002053. doi: 10.1371/journal.pmed.1002053.

3. Novo AM, Batista S. Multiple sclerosis: implications of obesity in neuroinflammation. Adv Neurobiol 2017;19:191-210.

4. Salem R, Bamer AM, Alschuler KN, Johnson KL, Amtmann D. Obesity and symptoms and quality of life indicators of individuals with disabilities. Disabil Health J 2014;7:124-130.

5. Cambil-Martín J, Galiano-Castillo N, Muñoz-Hellín E, Díaz-Rodríguez L, Laguarta-Val S, Fernández-de-Las-Peñas C, Arroyo-Morales M. Influence of body mass index on psychological and functional outcomes in patients with multiple sclerosis: a cross-sectional study. Nutr Neurosci 2016;19:79-85.

6. Tettey P, Balogun S. Obesity and multiple sclerosis: prevalence, susceptibility and progression in disability. Future Neurol 2017;12:149-157.

7. Russell RD, Langer-Gould A, Gonzales EG, Smith JB, Brennan V, Pereira G, Lucas RM, Begley A, Black LJ. Obesity, dieting, and multiple sclerosis. Mult Scler Relat Disord 2019;39:101889. doi: 10.1016/j.msard.2019.101889. Epub ahead of print.

8. Cameron MH, Nilsagard Y. Balance, gait, and falls in multiple sclerosis. Handb Clin Neurol 2018;159:237-250.

9. Bethoux F. Gait disorders in multiple sclerosis. Continuum (Minneap Minn) 2013;19(4 Multiple Sclerosis):1007-1022

10. Pilutti LA, Dlugonski D, Pula JH, Motl RW. Weight status in persons with multiple sclerosis: implications for mobility outcomes. J Obes 2012;2012:868256. doi: 10.1155/2012/868256

11. Kalron A. Relationship of obesity with gait and balance in people with multiple sclerosis. Am J Phys Med Rehabil 2017;96:140-145.

12. Sebastião E, Motl RW. Body mass index and cardiorespiratory fitness in persons with multiple sclerosis. Acta Neurol Scand 2018;138:315-319.

13. Schetz M, De Jong A, Deane AM, Druml W, Hemelaar P, Pelosi P, Pickkers P, Reintam-Blaser A, Roberts J, Sakr Y, Jaber S. Obesity in the critically ill: a narrative review. Intensive Care Med 2019:45:757-769.

14. Pilutti LA, Motl RW. Body Mass Index underestimates adiposity in persons with multiple sclerosis. Arch Phys Med Rehabil 2016;97:405-412.

15. Thompson AJ, Banwell BL, Barkhof F, Carroll WM, Coetzee T, Comi G, Correale J, Fazekas F, Filippi M, Freedman MS, Fujihara K, Galetta SL, Hartung HP, Kappos L, Lublin FD, Marrie RA, Miller AE, Miller DH, Montalban X, Mowry EM, Sorensen PS, Tintoré M, Traboulsee AL, Trojano M, Uitdehaag BMJ, Vukusic S, Waubant E, Weinshenker BG, Reingold SC, Cohen JA. Diagnosis of multiple sclerosis: 2017 revisions of the McDonald criteria. Lancet Neurol 2018;17:162-173

16. Kurtzke JF. Rating neurologic impairment in multiple sclerosis: an expanded disability status scale (EDSS). Neurology 1983;33:1444-14452.

17. Kieseier BC, Pozzilli C. Assessing walking disability in multiple sclerosis. Mult Scler 2012;18:914-924.

18. Paltamaa J, West H, Sarasoja T, Wikström J, Mälkiä E. Reliability of physical functioning measures in ambulatory subjects with MS. Physiother Res Int 2005;10:93-109. Erratum in: Physiother Res Int 2006;11:123. 
19. Kalron A, Dolev M, Givon U. Further construct validity of the Timed Up-andGo Test as a measure of ambulation in multiple sclerosis patients. Eur J Phys Rehabil Med 2017;53:841-847.

20. Hobart JC, Riazi A, Lamping DL, Fitzpatrick R, Thompson AJ. Measuring the impact of MS on walking ability: the 12-Item MS Walking Scale (MSWS-12). Neurology 2003;60:31-36.

21. Dib H, Tamam Y, Terzi M, Hobart J. Testing patient-reported outcome measurement equivalence in multinational clinical trials: an exemplar using the 12-item multiple sclerosis walking scale. Mult Scler J Exp Transl Clin 2017;3:2055217317728740. doi: 10.1177/2055217317728740.

22. Gültekin T, Ozer BK, Akin G, Bektaş $Y$, Sağir M, Güleç E. Prevalence of overweight and obesity in Turkish adults. Anthropol Anz 2009;67:205-212.

23. Marrie RA. Comorbidity in multiple sclerosis: implications for patient care. Nat Rev Neurol 2017;13:375-382

24. Marck CH, Neate SL, Taylor KL, Weiland TJ, Jelinek GA. Prevalence of Comorbidities, overweight and obesity in an international sample of people with multiple sclerosis and associations with modifiable lifestyle factors. PloS One 2016;11:e0148573. doi: 10.1371/journal.pone.0148573.

25. Mafort TT, Rufino R, Costa CH, Lopes AJ. Obesity: systemic and pulmonary complications, biochemical abnormalities, and impairment of lung function. Multidiscip Respir Med 2016;11:28.

26. Stampanoni Bassi M, lezzi E, Buttari F, Gilio L, Simonelli I, Carbone F, Micillo T, De Rosa V, Sica F, Furlan R, Finardi A, Fantozzi R, Storto M, Bellantonio P, Pirollo P, Di Lemme S, Musella A, Mandolesi G, Centonze D, Matarese G. Obesity worsens central inflammation and disability in multiple sclerosis. Mult Scler 2020;26:1237-1246.

27. Liparoti M, Della Corte M, Rucco R, Sorrentino P, Sparaco M, Capuano R, Minino R, Lavorgna L, Agosti V, Sorrentino G, Bonavita S. Gait abnormalities in minimally disabled people with multiple sclerosis: a3D-motion analysis study. Mult Scler Relat Disord 2019;29:100-107.
28. Ayan H, Altin Ertekin Ö, Kahraman T, Ozakbas S. Balance and gait impairment in persons with multiple sclerosis with the absence of clinical disability. Turk J Neurol 2020;26:224-229.

29. Moon Y, McGinnis RS, Seagers K, Motl RW, Sheth N, Wright JA, Jr., Ghaffari R, Sosnoff JJ. Monitoring gait in multiple sclerosis with novel wearable motion sensors. PloS One 2017;12:e0171346. doi: 10.1371/journal.pone.0171346.

30. Meng H, O'Connor DP, Lee BC, Layne CS, Gorniak SL. Alterations in overground walking patterns in obese and overweight adults. Gait Posture 2017:53:145-150.

31. Manawat R, Shweta S. Effect of six-minute walk test in obesity. Int J Med Sci Public Health 2018:7:260-263.

32. Halabchi F, Alizadeh Z, Sahraian MA, Abolhasani M. Exercise prescription for patients with multiple sclerosis; potential benefits and practical recommendations. BMC Neurol 2017;17:185.

33. Motl RW, Sandroff BM, Kwakkel G, Dalgas U, Feinstein A, Heesen C, Feys P Thompson AJ. Exercise in patients with multiple sclerosis. Lancet Neurol 2017;16:848-856.

34. Mokhtarzade M, Agha-Alinejad H, Motl RW, Negaresh R, Baker JS, Zimmer P. Weight control and physical exercise in people with multiple sclerosis: current knowledge and future perspectives. Complement Ther Med 2019;43:240-246.

35. Pilutti LA, Dlugonski D, Sandroff BM, Klaren RE, Motl RW. Internet-delivered lifestyle physical activity intervention improves body composition in multiple sclerosis: preliminary evidence from a randomized controlled trial. Arch Phys Med Rehabil 2014;95:1283-1288

36. Fonseca-Junior SJ, Sá CG, Rodrigues PA, Oliveira AJ, Fernandes-Filho J. Physical exercise and morbid obesity: a systematic review. Arq Bras Cir Dig 2013;26(Suppl 1):67-73. 